\title{
ESTUDO DA CAPACIDADE DO PROCESSO DE PRENSAGEM E ENSAIOS DE FLEXÃO DE BRIQUESTES FEITOS DE ARGILA
}

\author{
Bruna Tereza de Oliveira Rocha - FIC/UNIS \\ Juliana Karine de Moraes Pereira - FIC /UNIS \\ Natália Aparecida de Carvalho Mariquito - FIC/UNIS \\ Tiago Bittencourt Nazaré - FIC/UNIS
}

\begin{abstract}
RESUMO: $O$ controle da qualidade vem sendo pesquisado progressivamente em todas as áreas da empresa, a fim de garantir competitividade no mercado. O objetivo e meta do presente artigo é analisar a prensagem da Argila, transformando-as em Briquetes, realizando ensaios de flexão e identificando os índices encontrados. $O$ intuito é analisar os mesmos no gráfico de controle e verificar a capacidade do processo com o auxílio dos índices Cp (mede a capacidade e quantifica a difusão obtida pela técnica que mede a real separação do processo) e Cpk (indica a separação entre a média do processo e o limite de identificação mais próximo), onde quanto maior forem esses índices, maior será a capacidade do processo em atender as especificações do produto, com a finalidade de melhoria do processo estudado. Desta forma, foi constatado, após análise, que o processo não atende as especificações esperadas para o produto.
\end{abstract}

Palavras-Chave: Prensagem, argila, capacidade do processo, briquetes.

\section{INTRODUÇÃO}

Confome Furtado e Silva (2018) As técnicas estatísticas são consideradas ferramentas fundamentais para o monitoramento da qualidade. A utilização destes mecanismos estatísticos no controle da qualidade gera resultados sólidos e consistentes no que diz respeito ao controle das variáveis.

Ainda segundo o autor acima o controle da qualidade como se conhece hoje somente manifestou-se com a Segunda Guerra Mundial, com a criação dos inspetores de qualidade dos produtos que checavam produto por produto, mas esse método foi logo substituído pelas “Técnicas Estatísticas de Controle da Qualidade".

De acordo com Rocha, Suarez, Guimarães (2014) O estudo feito tem grande importância na fabricação de produtos que utilizam a argila como matéria prima, pois demonstra a relevância do peneiramento do material antes de iniciar o processo de produção, pois sem esse procedimento o produto final pode sofrer alterações.

Para Gonçalez e Werner (2009) Devido às diferenças encontradas nos resultados obtidos após a prensagem e os ensaios de flexão dos materiais, surge a necessidade de analisar e identificar através do estudo da capacidade do processo para cumprir adequadamente com o propósito de informar que total de produtos do método irão atender os critérios estimados.

Segundo Barreto, Rocha e Junior (2016) O presente artigo identificou os índices de 
capacidade potencial do processo, $\mathrm{Cp}$ e Cpk através da prensagem da argila a fim de encontrar dados para uma análise por meio de testes com auxílio do software MINITAB que foi utilizado nos estudos do presente artigo.

O objetivo geral do presente artigo é verificar através dos briquetes quais desses índices encontrados estão fora de controle e verificar se o processo estudado atende e possui capacidade de produzir peças dentro das especificações esperadas para o produto. E o objetivo específico é de analisar a Capacidade do Processo através do índice "Cp" e "Cpk".

\section{REVISÃO DE LITERATURA}

\subsection{Controle da Qualidade}

Segundo Shissatti (1993 apud Juran, 2009) fiscalizar um processo é sobre tudo controlar a qualidade deste. O controle da qualidade como método gerencial constituído pelas etapas: avaliação do desempenho real da qualidade, comparação do desempenho real com suas metas e atuação nas diferenças entre desempenho real e metas. O conceito de controle da qualidade é permanecer o status em seu modo planejado de forma que atinja as metas planejadas. Os níveis de qualidade, ou propósitos definidos para um processo é o estímulo para o controle.

De acordo com Campos (2004 apud Hastenreiter, 2010) o TQC no modelo japonês pode ser estabelecido como um conjunto gerencial, que por modo da relação de todas as pessoas em todas as áreas da empresa, visa atender sua carência através do controle da qualidade, propiciando produção e serviços a condições mais econômicas. O TQC visa à satisfação do cliente para assegurar a sobrevivência da administração no mercado concorrencial. As partes interessadas são chamadas de stakeholders e apresentam estrita associação com a qualidade final obtida, conforme as necessidades e dos interesses de cada um.

Para Werkema (1995 apud Hastenreiter, 2010) os princípios básicos do Controle da Qualidade Total são:

Qualidade: A palavra qualidade pode ser entendida sob várias perspectivas. É necessário para que se estabeleça o controle da qualidade total a ser efetuado. Segundo Campos (2004 apud Hastenreiter, 2010) diz que se tem um bem ou atividade de qualidade quando há auxilio máximo, verdadeiro, garantido e no prazo certo atendendo as necessidades do cliente.

Processo: Pode ser compreendido como um composto de entradas em um método 
(financeiro, pessoal e intelectual) que sentem transformações conduzidas por princípios internos e externos, tornando-se uma escapatória para o recurso ou operação realizada.

\subsection{Capacidade do Processo}

De acordo com Montgomery (2004 apud Oliveira et al., 2011) o diagnóstico da capacidade do método é uma prática que estima a versatilidade do recurso e concedendo uma comparação com os padrões do produto. A eficácia do processo consiste a sua semelhança na formação e define duas formas de considerar essa variabilidade, seja ao longo do tempo, seja prementemente.

Segundo Souza (2009 apud Siqueira et al., 2009) Capacidade do Processo é pertinente habilidade da forma de gerar resultados programados. Com o acréscimo da competência de um processo, torna-se provável e apreciável as causas mais importantes de baixa qualidade e produtividade, onde são monitoradas e descartadas. Ainda para Florac e Carleton (1999 apud Siqueira et al., 2009) a capacidade do Processo relata a possibilidade do desempenho de um procedimento sob controle estatístico.

\section{3 Índice Cp e Índice Cpk}

Segundo Spiring (1997 apud Lima et al., 2006) o índice de capacidade Cp tem como proporção comum medir a capacidade e quantifica a difusão obtida pela técnica que mede a real separação do processo. É uma grandeza de desempenho de aptidão do método.

De acordo com Cheng et al. (1998 apud Rennó, Oliveira, 2013) A eficácia do processo é habitualmente medida por moderado índices, cujo propósito é disponibilizar um código compartilhado e de simples compreensão para mensurar a atividade quanto a aptidão de gerar itens de acordo com uma estipulada flexibilidade.

Segundo Oliveira et al. (2011) o índice Cp conceitua que o sistema está centralizado no número nominal da especificação, determinado pela equação (1). LSE é o Limite Superior de Especificação; LIE é o Limite Inferior de Especificação e ơ é o desvio-padrão do processo.

Este índice confronta a variabilidade concedida ao sistema com a variabilidade autêntica do método. Contudo, quanto maior for o resultado do índice $\mathrm{Cp}$, maior será a Capacidade do Processo em atender as especificações. E ainda, segundo Montgomery (2004 apud Oliveira et al., 2011) para interpretar este índice, deve-se observar o Quadro 1. 
Quadro 1 - Intervalos de referência para análise do índice Cp.

$$
\mathrm{Cn}=\frac{L S E-L I E}{6 \sigma} \quad \text { Eq. (1) }
$$

\begin{tabular}{|c|c|c|}
\hline $\mathrm{Cp}$ & Itens não-conformes (PPM) & Interpretação \\
\hline $\mathrm{Cp}<1$ & Acima de 2.700 & Processo Incapaz \\
\hline $1 \leq \mathrm{Cp} \leq 1,33$ & 64 a 2700 & Processo aceitável \\
\hline $\mathrm{Cp} \geq 1,33$ & Abaixo de 64 & Processo potencialmente capaz \\
\hline
\end{tabular}

Fonte: Montgomery (2004)

De acordo com Kane (2000 apud Rennó, Oliveira, 2013) o índice Cpk, então indica a separação entre a média do processo e o limite de identificação mais próximo. $\mathrm{O}$ fato de que nem ao menos o processo obtém-se centralizado no valor nominal da caracterização, então a função Cp expõe-se a soluções incorretas. A proposição do índice de desempenho Cpk leva em argumentos a distância média do procedimento em correlação aos limites de particularização. No momento em que o centro ficar centralizado no valor nominal de caracterização, obtém-se que $\mathrm{Cp}=\mathrm{Cpk}$.

Segundo Oliveira et al. (2011) conforme na realidade nem sempre o processo está centralizado no valor nominal da especificação logo, o emprego do índice Cp é capaz de chegar a resultados incorretos. Segundo Kane (1986 apud Oliveira et al., 2011) informou o índice Cpk, que conceitua o intervalo da média do sistema em correspondência aos limites de especificações. Este índice é encontrado pela Equação (2).

LSE é o Limite Superior de Especificação, LIE é o Limite Inferior de Especificação, u é a Média do processo e ơ é o Desvio-padrão do processo.

$$
C n k=\operatorname{MIN}\left(\frac{\operatorname{LSE}-\mu}{3 \sigma}: \frac{\mu-\text { LIE }}{3 \sigma}\right) . \quad \text { Eq. (2) }
$$

Boyles (1991 apud Rennó, Oliveira, 2013) aponta que o índice Cpk não demonstra perfeitamente a concentração do processo. Com isso, o autor utiliza de técnicas gráficas onde por meio de comparações dos índices comprova que Cpk pode errar na discrepância de recurso fora do ponto e dentro do ponto.

De acordo com Montgomery (2004 apud Oliveira et al., 2011) entre os meios estatísticos 
gastos para analisar se um método está empregado de acordo com os princípios, ressaem-se os índices de competência que tem como origem aperfeiçoar-se a proporção de peças ou produtos defectivos produzidos. O $\mathrm{Cp}$ e $\mathrm{Cpk}$ foram principais índices expressados sendo o mais importante e comum na indústria.

\section{METODOLOGIA}

\subsection{Materiais}

Para a produção da prática foram utilizados os seguintes materiais e equipamentos:

- Argila (Embalado por Argigoma);

- Recipientes para armazenamento da Argila;

- Balança Eletrônica BG400;

- Prensa Hidráulica RIBEIRO 15 TON;

- Paquímetro Worker 150 mm 6 Pol;

- Máquina de Ensaio OSWALDO FILIZOLA AME - 2 KN;

- Software Dynaview Standard M versão 2.6.5;

- Software Excel 2010;

- Software MINITAB, versão 16.

\subsection{Métodos}

As pesquisas e o levantamento das informações sobre o tema foram feitos através de pesquisas em artigos relacionados ao tema.

No presente trabalho foi determinada a capacidade de um processo através da prensagem da "Argila". Segundo Oliveira (2017) na prensagem, o material permanece em prática da força de compressão em baixa velocidade e a pressão alcança seu valor máximo pouco antes de retirados, de forma que as camadas mais profundas da estrutura do material são obtidas no procedimento de conformação. Os dados foram obtidos a partir da prensagem do material, no laboratório de materialografia das Faculdades Integradas de Cataguases - Grupo Unis, realizados nos meses de Agosto e Setembro de 2017.

O procedimento primário praticado foi separar uma quantidade do material em um recipiente a fim de utilizá-lo como amostra para o estudo. Devido à limitação do Laboratório de 
materialografia da FIC o material não foi peneirado. Após esse passo, o material foi pesado com a balança eletrônica BG400 e separados 30 amostras em recipientes diferentes, tendo cada uma com 40 gramas. De acordo com Saleme (2000) a briquetagem compreende-se na utilização de compressão de uma massa de partículas, junto ou sem acréscimo de ligante, e com ou sem o tratamento térmico. O segundo passo foi a produção de briquetes de argila através da prensagem. Logo após a prensagem dos Briquetes, todas as amostras foram pesadas (g) novamente. Com os pesos de cada amostra em mãos, foi feito um Gráfico de Controle com o software MINITAB, para verificar se algum briquete estava fora de controle, mas todos estavam dentro dos Limites de Controle. Logo após esse passo, sua altura $(\mathrm{mm})$ e diâmetro $(\mathrm{mm})$ foram medidos através do paquímetro e pelo software Dynaview Standard foi calculado sua área de secção $\left(\mathrm{mm}^{2}\right)$ e feito os ensaios de flexão em todas as amostras a fim de encontrar a Máxima Força (N), Deformação (mm) e máxima Tensão (Mpa), conforme dados demostrados nos quadros 2, 3 e 4 . Com os dados em planilha no software Excel, estes foram transferidos para o MINIT AB e novamente realizado um gráfico de controle para verificar se existe alguma amostra fora dos limites de controle.Após teste com o Gráfico de Controle, foi constatado que nenhuma das amostras saiu dos Limites de Especificação e foram utilizadas todas as amostras para verificar a Capabilidade do processo.

Figura 1 - Briquetes após prensagem.

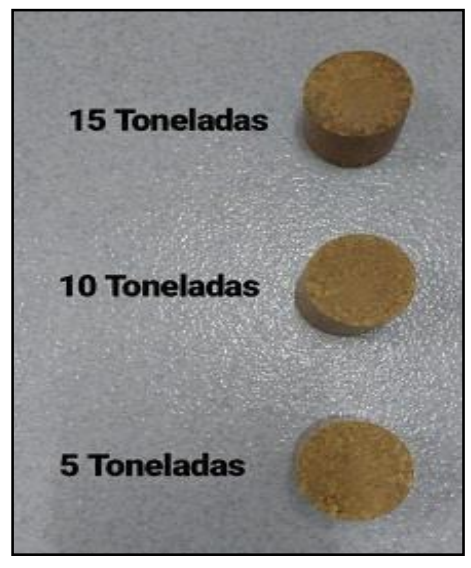

Fonte: Autor (2017).

Na Figura 1, pode-se observar 3 briquetes após a prensagem, com as pressões indicadas ao lado de cada amostra. 
Quadro 2 - Dados obtidos após a prensagem com 5 toneladas e flexão dos Briquetes.

\begin{tabular}{|c|c|c|c|c|c|c|c|}
\hline \multicolumn{7}{|c|}{$\mathbf{5}$ toneladas } \\
\hline Amostra & Peso (grs.) & Altura (mm) & Diâmetro (mm) & Área Secção (mm $\mathbf{~})$ & Máxima Força (N) & Deformação (mm) & Máximo Tensão (MPa) \\
\hline 1 & 35,525 & 25 & 30 & 706,85 & 16,9 & $-3,9347$ & 0,024 \\
\hline 2 & 35,317 & 25 & 30 & 706,85 & 16,9 & $-3,9347$ & 0,024 \\
\hline 3 & 35,543 & 25,2 & 30 & 706,85 & 15,4 & $-2,6678$ & $-0,218$ \\
\hline 4 & 36,042 & 25 & 30,2 & 716,31 & 16,7 & $-13,0715$ & 0,022 \\
\hline 5 & 35,977 & 24,65 & 30 & 706,85 & 16,8 & $-14,27$ & 0,024 \\
\hline 6 & 35,818 & 25,35 & 30,3 & 721,06 & 16,7 & $-15,8067$ & 0,023 \\
\hline 7 & 36,474 & 24,7 & 30 & 706,85 & 16,5 & $-13,7568$ & 0,022 \\
\hline 8 & 37,646 & 25,6 & 30,2 & 716,31 & 16,9 & $-15,8464$ & 0,024 \\
\hline 9 & 36,835 & 24,7 & 30,2 & 716,31 & 16,9 & $-15,1376$ & 0,024 \\
\hline 10 & 36,768 & 25 & 30 & 706,85 & 16,8 & $-15,4664$ & 0,024 \\
\hline
\end{tabular}

Fonte: Autor (2017).

No quadro 2 demonstra que a amostra 3, na coluna da Máxima Tensão (MPa), obteve um resultado muito diferente das demais amostras, e com isso, demonstra a importância do peneiramento do material antes da prensagem e flexão dos briquetes, pois com o peneiramento o material fica mais concentrado e com menos resíduos não conformes.

Quadro 3 - Dados obtidos após a prensagem com 10 toneladas e flexão dos Briquetes.

\begin{tabular}{|c|c|c|c|c|c|c|c|}
\hline \multicolumn{8}{|c|}{10 toneladas } \\
\hline Amostra & Peso (grs.) & Altura (mm) & Diâmetro (mm) & Área Secção $\left(\mathrm{mm}^{2}\right)$ & Máxima Força (N) & Deformação (mm) & Máximo Tensão (MPa) \\
\hline 1 & 36,2 & 23,15 & 30 & 706,85 & 16,9 & $-13,1263$ & 0,024 \\
\hline 2 & 36,433 & 23,5 & 30,2 & 716,31 & 16,9 & $-15,4698$ & 0,024 \\
\hline 3 & 36,156 & 23,5 & 30 & 706,85 & 17 & $-16,1408$ & 0,024 \\
\hline 4 & 36,227 & 24 & 30,2 & 716,31 & 15,4 & $-6,935$ & 0,021 \\
\hline 5 & 36,415 & 23,55 & 30 & 706,85 & 16,9 & $-16,5405$ & 1,121 \\
\hline 8 & 36,712 & 23,65 & 30 & 706,85 & 0,3 & $-9,4882$ & 0 \\
\hline 9 & 37,597 & 24,4 & 30 & 706,85 & 0 & 0 & $-14,9397$ \\
\hline 10 & 36,684 & 23,8 & 30 & 706,85 & 757,6 & $-16,65$ & 1.072 \\
\hline
\end{tabular}

Fonte: Autor (2017).

No Quadro 3, as amostras 8, 9 e 10 na coluna de Máxima Força (N) demonstrou um resultado fora do padrão das demais amostras, evidenciando a existência de itens não conformes juntamente ao material analisado. 
Quadro 4 - Dados obtidos após a prensagem com 15 toneladas e flexão dos Briquetes.

\begin{tabular}{|c|c|c|c|c|c|c|c|}
\hline \multicolumn{8}{|c|}{15 toneladas } \\
\hline Amostra & Peso (grs.) & Altura (mm) & Diâmetro (mm) & Área Secção $\left(\mathrm{mm}^{2}\right)$ & Máxima Força (N) & Deformação (mm) & Máximo Tensão (MPa) \\
\hline 1 & 34,676 & 22,3 & 30 & 706,85 & 0,4 & $-22,4$ & 0,001 \\
\hline 2 & 34,553 & 22,35 & 30 & 706,85 & 0,4 & $-16,0504$ & 0,001 \\
\hline 4 & 33,186 & 21 & 30 & 706,85 & 608,9 & $-21,48$ & 0,861 \\
\hline 5 & 34,658 & 22,4 & 30 & 706,85 & 0,4 & $-9,603$ & 0,001 \\
\hline 8 & 33,93 & 22 & 30 & 706,85 & 0,3 & $-14,1$ & 0 \\
\hline 9 & 35,686 & 22,7 & 30 & 706,85 & 0,2 & $-23,54$ & 0 \\
\hline 10 & 35,102 & 22,65 & 30 & 706,85 & 0,3 & $-0,3$ & 0 \\
\hline
\end{tabular}

Fonte: Autor (2017).

No Quadro 4, as amostras 4 e 6 possuem valores da Máxima Força (N) muito desproporcional aos resultados, das demais amostras, e com isso, compreende-se que ao efetuar o ensaio de flexão, os briquetes possuem elementos não conformes, ocasionado pelo não peneiramento.

Para a determinação da capacidade do processo foram identificados os Índices Cp e Cpk. Portanto, para estabelecer o índice Cp, devem-se seguir os seguintes passos: Coletar os dados a partir dos trabalhos realizados no laboratório mencionado anteriormente; Tabular os dados e determinar o Gráfico de controle $\bar{X}$ e R, utilizando o software Minitab;

Para determinar o índice Cpk, devem-se prosseguir as seguintes etapas: Coletar os dados a partir dos trabalhos realizados no laboratório mencionado anteriormente; Tabular os dados e determinar o Gráfico de controle $\bar{X}$ e R, utilizando o software Minitab; Encontrar o desvio padrão utilizando a fórmula: $\sigma=\frac{R}{\mathrm{~d} 2}$ Aplicar a fórmula $\mathrm{Cp}=\frac{L S E-L I E}{6 \sigma \mathrm{I}} \quad$ Determinar a média do processo $(\mu)$ através da d2 soma dos valores das amostras, dividido pelo número de amostras analisadas; Aplicar a fórmula $C p k=\operatorname{MIN}\left(\frac{\mathrm{LSE}-\mu}{3 \sigma} ; \frac{\mu-\mathrm{LIE}}{3 \sigma}\right)$.

Em seguida, após determinação dos índices Cp e Cpk, utilizará Quadro 1, mencionado na Revisão de Literatura, para interpretar os resultados obtidos de Cp e Cpk, e assim, verificar se o produto possui capacidade para atender as especificações esperadas para o mesmo.

\section{RESULTADOS E DISCUSSÕES}

Com os dados obtidos foi possível gerar três quadros de informações com todas as 
amostras utilizadas em três pressões diferentes e encontrar a Capacidade do processo através dos índices Cp e Cpk calculados no software MINITAB.

Os resultados após a prensagem dos Briquetes estão descriminados no Gráfico de Controle informado na Figura 2, com base nos pesos (g) encontrados nestes após sua prensagem, onde pode se observar que nenhumas das amostras estão fora de Controle e, com isso, foi feito à sequência do desenvolvimento com todas as amostras.

Figura 2 - Gráfico de Controle após a prensagem dos Briquetes.

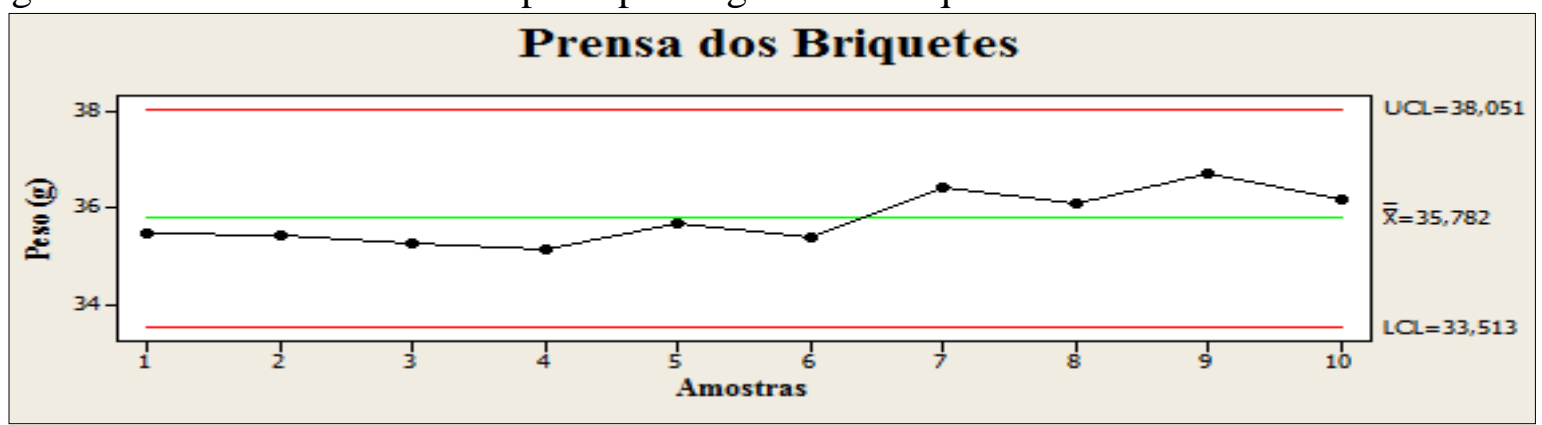

Fonte: Autor (2017).

Na figura 3, foi feito um Gráfico de Controle com todas as amostras após a realização do Ensaio de Flexão dos Briquetes, analisando a Força máxima feita pela máquina e foi constatado que nenhuma das amostras saiu de Controle.

Figura 3 - Gráfico de Controle após o Ensaio de Flexão dos Briquetes.

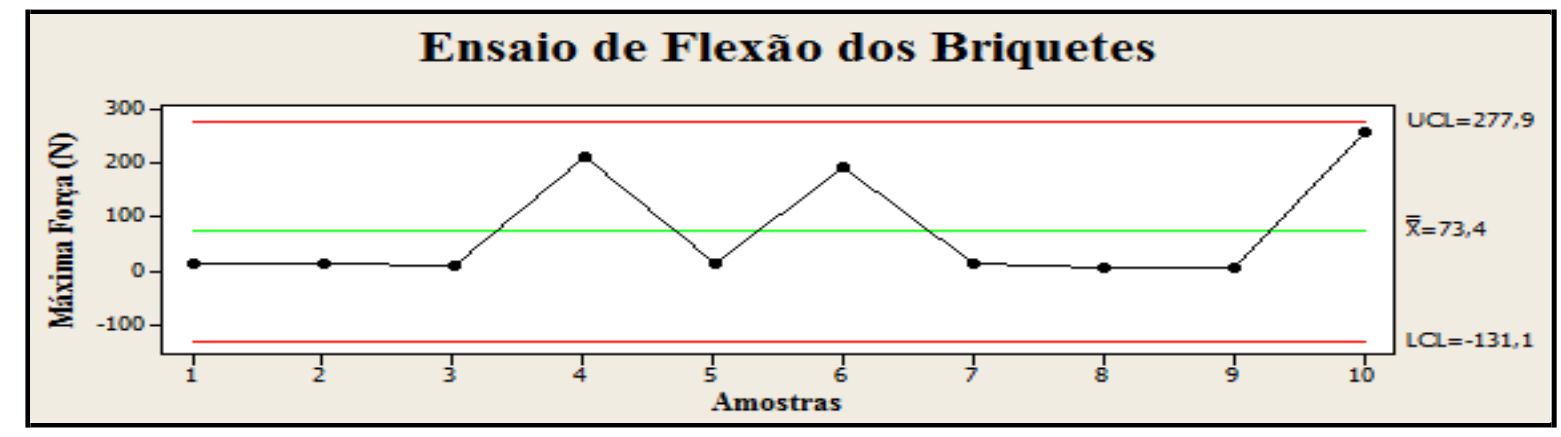

Fonte: Autor (2017).

Os valores da Capabilidade do processo encontrado na figura 4 foram de: $\mathrm{Cp}=0,58 \mathrm{e}$ $\mathrm{Cpk}=0,58$. Esses valores obtidos evidenciaram que o processo é Incapaz, ou seja, não atende e não possui capacidade de produzir peças dentro das especificações esperadas para o produto. 
Figura 4 - Gráfico da Capabilidade do Processo após a quebra dos Briquetes

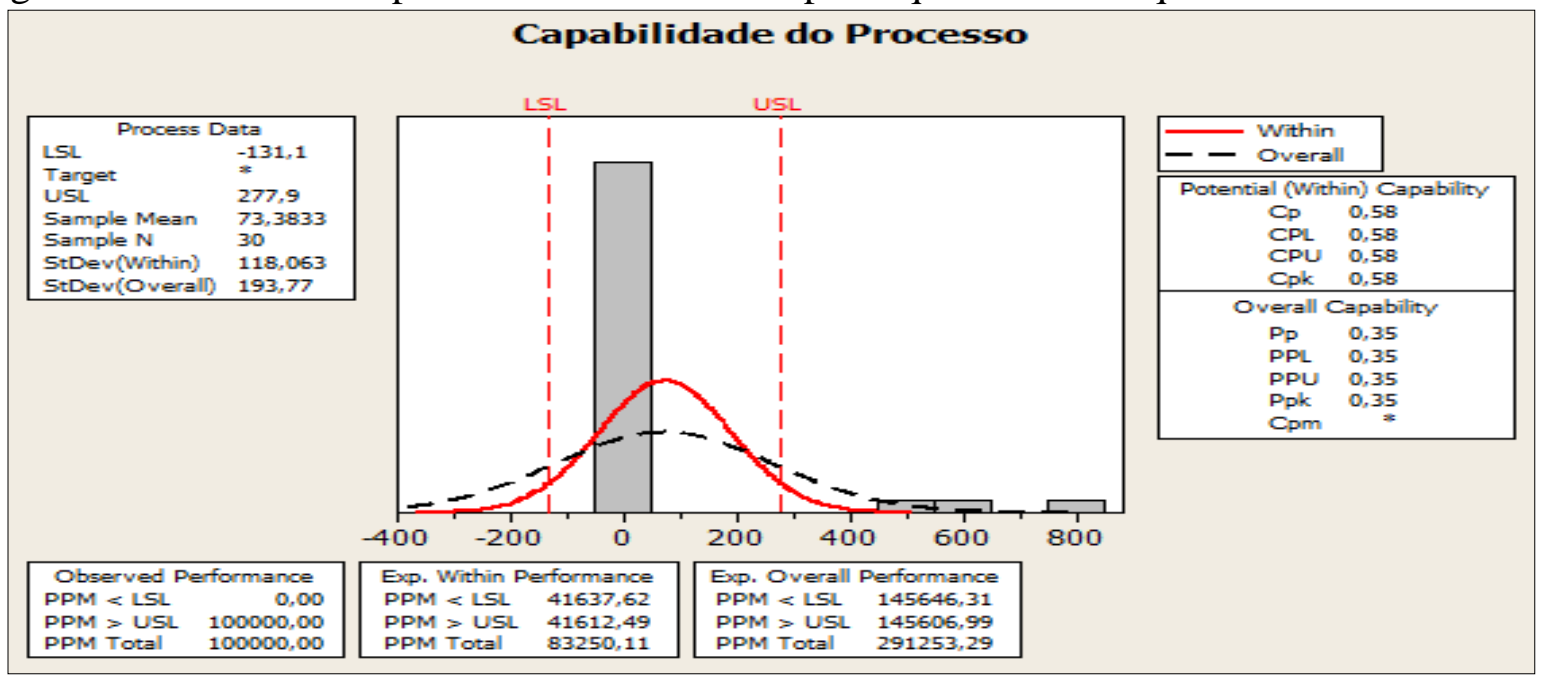

Fonte: Autor (2017).

A figura 4, além de informar os índices Cp e Cpk, também demonstra os valores da Capacidade Geral do Processo, LSC, LIC, entre outros índices importantes que se deve levar em consideração na produção desse produto.

Segundo CETEM (2004) o peneiramento tende-se a obter o material com as partículas mais concentradas e com o fracionamento da mesma em duas ou mais partes, com fragmentos de medidas diferentes, possibilitando obter as amostras com maior qualidade e com menos resíduos não conformes, prejudicando os resultados analisados após o processo praticado.

\section{CONCLUSÃO}

A pesquisa feita tem grande relevância no Controle da Qualidade dos produtos, onde foi feito o estudo da Capacidade de um processo através da prensagem e flexão das amostras de argila com demonstrações dos resultados em gráficos de controle com parâmetros dos resultados obtidos dos índices Cp e Cpk nos intervalos de referência para análise do índice Cp elaborado por Montgomery (2004).

A metodologia utilizada, apesar do material não ter sido peneirado, foi de grande importância para a obtenção dos resultados do estudo da Capabilidade do processo, pois foi recolhido o material a ser analisado, pesado separadamente cada amostra com pesos iguais, feito à prensagem isoladamente das mesmas com as três pressões analisadas e feito uma nova pesagem dos briquetes obtidos, logo após esses passos, aconteceu os ensaios de flexão de todos 
os briquetes e anotados todos os dados obtidos nos quadros 2, 3 e 4 . E, tanto na prensagem quanto nos ensaios de flexão das amostras os dados obtidos foram analisados em gráfico de controle e não tiveram nenhum corpo de prova que saiu dos limites de controle.

Contudo, o processo não atendeu as especificações do material, pois os valores obtidos da Capacidade desse processo foram de $\mathrm{Cp}=0,58 \mathrm{e} \mathrm{Cpk}=0,58$, apresentando um processo incapaz e que não possui capacidade de produzir peças dentro das especificações esperadas para esse produto. $\mathrm{O}$ não peneiramento do material pode ter sido um dos fatores que prejudicou os índices Cp e Cpk, pois a Argila detinha de diversas substâncias não conformes que interferiu de uma forma negativa no resultado dos índices. Portanto, deverá ser feito um novo estudo com o peneiramento da argila antes de operar a prensagem, e os ensaios de flexão da mesma, pois desta forma as amostras do material propende-se a ter suas partículas mais concentradas, e deste modo, poderá conseguir um produto final com maior qualidade e com melhores índices da capacidade do processo.

\section{REFERÊNCIAS BIBLIOGRÁFICAS}

BOYLES, R. A. The Taguchi Capability Index. Journal of Quality Technology, volume 23, 17-26, (1991). In: RENNÓ, Luciana; OLIVEIRA, Edson. A utilização da função perda de taguchina prática do controle estatístico de processo. Itajubá - MG: 1997, p.4.

BARRETO, R. R.; ROCHA, O. R.; JUNIOR, A. B Análise da capacidade de um processo de revestimento de bobinas Encontro Nacional de Engenharia de Produção, Belo Horizonte, 2011.

CAMPOS, V. F. TQC: Controle da qualidade total (no estilo japonês). 8 ed. Nova Lima : INDG Tecnologia e Serviços Ltda, 2004. 256p. In: HASTENREITER, Juliana. Aplicação do controle estatístico do processo no envase de leite UHT em uma indústria de Laticínios. Juiz de Fora - MG: 2010, p. 24 - 25.

CETEM (Centro de Tecnologia Mineral Ministério da Ciência e Tecnologia). Disponível

em: http://www.ufjf.br/baccan/files/2012/11/Cap-5-Peneiramento.pdf. Acesso em: 26/09/2017. p. 2.

CHAN, L.K., CHENG, S.W., SPIRING, F.A.A New Measure of Process Capability: Cpm. Journal of Quality Technology, volume 20, 162-175, (1988). In: RENNÓ, Luciana; OLIVEIRA, Edson. A utilização da função perda de taguchina prática do controle estatístico de processo. Itajubá - MG: 1997, p. 3 - 4.

DOUGLAS R. B. FURTADO; e STEPHANIE C. A. da Silva. (Gestão da Qualidade) http://gestao-dequalidade.info/fale-conosco.html. Acesso:10/04/2019

JURAN, J. M. Juran na Liderança pela Qualidade. 3a Edição. Livraria Pioneira Editora, São Paulo, 1993. In: CORRÊA, Jairo; NETO, Anselmo. Estudo Do Controle e Análise Da Capacidade Do Processo De Produção De Água Potável. Curitiba - PR: 2009, p. 1415 - 1416.

KANE, V.E. Process Capability Indices Journal of Quality Technology, v. 18, n. 1, 41-52, (1986).In: PEDRINI, Danilo; SCHWENGBER, Carla. Índices de capacidade do processo: proposta baseada em modelos de regressão e um fluxograma orientativo. Bento Gonçalves - RS: 2009, p.4.

MONTGOMERY, Douglas C. Introdução ao Controle Estatístico da Qualidade. $4^{\mathrm{a}}$ ed., LTC (2004). In: OLIVEIRA, J. B.; SOUTO, R. R.; MAIA, R. D. A.; MEIRA, J. A.; LIMA, V. S. P. Análise da capacidade de

um processo: Um estudo de caso baseado nos indicadores Cp e Cpk. XXXI Encontro Nacional de Engenharia de Produção, Belo Horizonte, 2011, p. 4 - 5.

OLIVEIRA, J. B.; SOUTO, R. R.; MAIA, R. D. A.; MEIRA, J. A.; LIMA, V. S. P.Análise da capacidade de um processo: Um estudo de caso baseado nos indicadores Cp e Cpk. XXXI Encontro Nacional de Engenharia de Produção, Belo Horizonte, 2011. In: BARRETO, Rômulo; ROCHA, Hélcio; JUNIOR, Cyro. Análise da capacidade 
de um processo de revestimento de bobinas de aço. Rio de Janeiro - RJ, 2016, p.3.

OLIVEIRA, BRUNO. (Upload Your Documents) Processo de forjamento por prensagem, 2017. Disponível em: https://pt.scribd.com/document/357333312/Forjamento-Por-Prensagem. Acesso em 12/05/2019.

SALEME, J. E. F. Estudo básico para briquetagem do carvão vegetal. Ouro Preto, Escola de Minas e Metalurgia, s.d. 19p. In: FERREIRA, Waldir e BRITO, José. Características e Índice de Combustão de Briquetes de Carvão Vegetal. São Paulo, 1991, p. 3.

SOUZA, F.S., PEDRINI, D.C. e CATEN, C.S. Índices de capacidade do processo: Comparação entre índices tradicionais e índices para gráficos de controle de regressão. XVI Simpósio de Engenharia de Produção (SIMPEP) 2009, Bauru, São Paulo - SP. In: PEDRINI, Danilo; SCHWENGBER, Carla. Índices de capacidade do processo: proposta baseada em modelos de regressão e um fluxograma orientativo. Bento Gonçalves - RS: 2009, p. 4 - 5 .

ROCHA, F. N.; SUAREZ, P. A. Z.; GUIMARÃES, E. M. Argilas e suas Aplicações em Utensílios e Materiais Cerâmicos. Rev. Virtual Quim., 2014, 6 (4), 1105-1120. Data de publicação na Web: 12 de junho de 2014.

WERKEMA, M. C. C. Ferramentas estatísticas básicas para o gerenciamento de processos. Belo Horizonte: Fundação Christiano Ottoni, v.2, 1995. In: HASTENREITER, Juliana. Aplicação do controle estatístico do processo no envase de leite UHT em uma indústria de Laticínios. Juiz de Fora - MG: 2010, p. 26 - 27.

WERKEMA, C; Ferramentas Estatísticas Básicas do Lean Seis Sigma Integradas: PDCA e DMAIC. Ed: Elservier; 2014 - Rio de Janeiro, pag: 01 e 02. 